\title{
Significance of Endometrial Cells in Cervical Cytology (by Conventional Pap \& Manual Liquid Based Cytology) in Abnormal Uterine Bleeding Cases
}

\author{
Lopa Mudra kakoti ${ }^{1}$, Nandini N. Manoli ${ }^{1}$, Nandish S. Manoli ${ }^{2}$ \\ ${ }^{1}$ Department of Pathology, JSS medical college, A Constituent of JSS University, Mysore, India \\ ${ }^{2}$ Department of OBG, JSS Medical College, a constituent of JSS University, Mysore, India
}

Email address:

lopamudrakakoti@gmail.com (L. M. kakoti)

\section{To cite this article:}

Lopa Mudra kakoti, Nandini N. Manoli, Nandish S. Manoli. Significance of Endometrial Cells in Cervical Cytology (by Conventional Pap \& Manual Liquid Based Cytology) in Abnormal Uterine Bleeding Cases. Science Journal of Clinical Medicine. Special Issue: Latest Different Concepts of Gynaecology. Vol. 4, No. 4-1, 2015, pp. 6-10. doi: 10.11648/j.sjcm.s.2015040401.12

\begin{abstract}
Abnormal uterine bleeding is common gynaecologic complaint. Causes may vary with age, the most worrisome cause is malignancy of endometrium. No widely accepted screening test for endometrial carcinoma exists, but cervical cytology has been found to be of some use in detecting endometrial diseases. Guidelines from the 2001 Bethesda system, in addition to reporting of atypical glandular cells (AGC) and adenocarcinoma, requires the reporting of benign appearing endometrial cells in women aged above 40 years. Smears for cervical cytology are collected from 80 patients in the age group of 20-75 years with complaints of bleeding per vagina. Cyto-histological correlation is attempted in which D\&C or hysterectomy specimen is sent. Manual Liquid Based Cytology is strongly advocated as it improves sample quality by removing obscuring factors. Out of 80 abnormal uterine bleeding cases 52 showed endometrial cells (10 atypical glandular cells \& 42 benign endometrial cells) \& 39 had further diagnostic evaluation of endometrium. The results indicated an association between endometrial cells in cervical cytology with carcinoma in 6 cases(15.4\%), 1 case with complex hyperplasia with atypia $(0.03 \%)$, while remaining $84.6 \%$ had benign endometrial pathology. Hence we concluded that presence of atypical endometrial cells in all women \& benign endometrial cells in post menopausal women( $>40 \mathrm{yr})$ has considerable clinical implications \& further diagnostic evaluation for endometrial sampling is of utmost importance.
\end{abstract}

Keywords: Endometrium, pap smear, manual liquid based cytology

\section{Introduction}

Abnormal uterine bleeding (AUB) is a commonly encountered complaint in gynaecology outpatient department. Although the causes vary with age, the most worrisome cause of perimenopausal or postmenopausal bleeding per vagina is the malignancy of the endometrium.

In about $10 \%$ of cases AUB is associated with endometrial cancer, but it can be caused by many other conditions such as polyps, myomas, hormonal dysfunction and most commonly in post menopausal women atrophy. ${ }^{1,2,3}$

With changing life style, increasing women life expectancy and drastic reduction of historically commoner cervical cancer by the successful implementation of pap smear screening programs, the increasing incidence of endometrial carcinoma is becoming an important challenge.
Though various methods have been introduced to know the endometrial status, no widely accepted screening test for endometrial carcinoma exists because of certain issues like cost-efffectiveness and patients acceptance. ${ }^{4,5}$

Endometrial cells are present incidentally on many pap tests, providing cytopathologists an oppertunity to examine these cells in specimen that may have been obtained for other reasons. ${ }^{6}$

Endometrial cells are a normal component of smear in first half of menstrual cycle when endometrium breakdown and shedding occurs. However, if endometrial cells are observed in smears in second half of cycle, the pathological significance is greater although cell shedding at this time can be associated with use of OCP, IUCD or use of HRT. ${ }^{7}$

An association has been reported with presence of endometrial cells on cervical smears and clinically significant uterine lesions. Hence The Bethesda system 2001 has 
mandated multiple reporting categories meant to apply to endometrial cells. ${ }^{8}$

Guidelines from the 2001 Bethesda system suggested, in addition to reporting of atypical glandular cells (AGC) and adenocarcinoma, the reporting of benign appearing endometrial cells in women aged above 40 years is mandatory and a review of literature supports this recommendation. ${ }^{9}$

Recently liquid based cytology has become an alternative to conventional pap smears as it reduces the preparation of inadequate smears by removing the obscuring factors such as blood, mucus and overlapping cells, increasing the diagnostic accuracy. ${ }^{10,11}$

Some studies have shown more accurate diagnosis of glandular lesion. ${ }^{12,13}$

It reduces the screening time, resulting in greater efficiency of cytopathologist and the most important advantage is the opportunity to use the residual sample for additional ancillary techniques, such as immunocytochemistry, flow cytometry and molecular biology. Infact, the LBP method enables storage of a variable amount of cells.

However, the cost of most commercially available LBC system is prohibitively expensive for resource-limited settings. ${ }^{12,13}$ Trained individuals and periodic maintenance of the equipment are required.

Many workers like Alves et al. ${ }^{12}$ and Lee et al. ${ }^{13}$ have worked on an improvised manual method for preparation of liquid based cytology smears, for cervical cancer screening and found it comparable with CPS

The present study aimed to explore the role of cervical cytology by CPS and MLBC in the diagnosis of endometrial pathology in our set up and to correlate glandular cells of endometrial origin cases with histology

\section{Objectives}

1. To explore the role of cervical cytology in the diagnosis of endometrial diseases in all abnormal uterine bleeding cases

2. To estimate the presence of endometrial cells (atypical $\&$ benign looking) \& proportion of them associated with significant endometrial pathology

3. To quantify any differences in the underlying rate of significant pathological outcomes between conventional and manual liquid based cytology

\section{Materials and Methods}

Samples were collected using split- smear technique from patients with bleeding history in the age group of 20 to 70 years attending the gynaecology out-patient clinic at JSS Hospital, Mysore, prospectively from July 2012 to July 2014 for a period of two years. Manual Liquid Based Cytology was strongly advocated as it improves sample quality and reduces the likelihood of false negative results by removing obscuring factors.

Scrape smears were collected using plastic Ayre's spatula. The scrapings were first put into MLBC vial containing liquid fixative and later evenly spread onto a glass slide, and immediately fixed in ethyl-alcohol fixative $(95 \%$ ethyl alcohol). After fixation, smears were stained using conventional Pap stain.

\section{Statistical Analysis}

Statistical methods applied were Descriptives, Chi-square test and Crosstabs (Contingency table analysis. Apart from the above, diagnostic accuracy, sensitivity and specificity were calculated manually. All the statistical calculations were done through SPSS for windows (v 20.0). A $P$ value of less than .05 was considered statistically significant.

\section{Results}

During the study period, 52 Pap tests showed endometrial cells including $38 \mathrm{nEMCs}$ and 14 aEMCs with history of abnormal uterine bleeding. The mean age was 45.5 years (range, 20-75 years), with women being postmenopausal were 24 patients. Remaining 28 cases had irregular menstrual cycle in the form of polymenorrhea, menorrhagia or intermenstrual bleeding.

Abnormal uterine bleeding history was most commonly seen in age group of $40-49$ year.

Among 52 cases, histopathological correlation in the form of total hysterectomy and endometrial sampling. was obtained in 39 cases.

Among the 52 cases with endometrial cells, 46 were detected in conventional pap smear and 38 cases in manual liquid based cytology. Out of 46 cases of endometrial cells35 were benign and 11 were atypical cells in conventional pap smear. While in manual liquid based cytology 28 were benign and 10 were atypical endometrial cells out of 38 cases.

On follow up with histopathology there were 8 cases of malignancy, 1 case of pre cancerous lesion and others were benign pathology. Among benign cases maximum of 8 cases of leiyomyoma with proliferative endometrium, followed by 6 cases of simple hyperplasia without atypia and 4 cases of adenomyomatous polyp.

Among 14 atypical endometrial cell cases, 13 had histopathological correlation with 8 cases being malignant, 1 precancerous lesion and 4 cases of benign endometrial pathology. The malignant cases diagnosed were 4 cases as type 1 endometrial carcinoma followed by 3 cases of squamous cell carcinoma and 1 case of type 2 endometrial carcinoma. The remaining benign cases were 2 adenomyomatous polyp, 1 atrophic endometrium and 1 endometritis.

Benign endometrial cells did not show any malignant pathology on histopathology follow up.

When the results were analysed presence of endometrial cells in cervical cytology was found to be significant $(p<0.05)$ and on cross tabulation of CPC,MLBC and HPE showed better contingency with MLBC (0.572 V/s 0.556).

MLBC also showed better sensitivity and positive predictive value. 

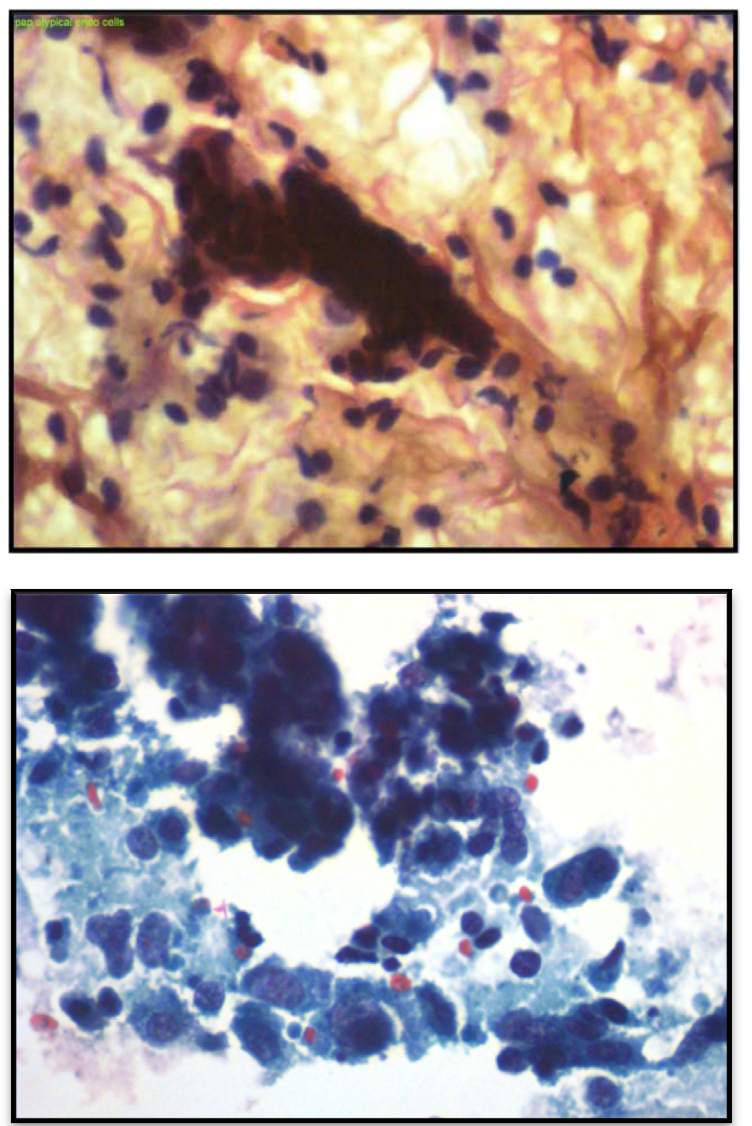

Fig. 1 \& 2. CPC (left) \& MLBC(right) (Pap, X400)-Atypical Glandular Cells of endometrial origin - Smear shows cells with high N/C ratio arranged in clusters with enlarged hyperchromatic nuclei with prominent nucleoli and fairly abundant cytoplasm

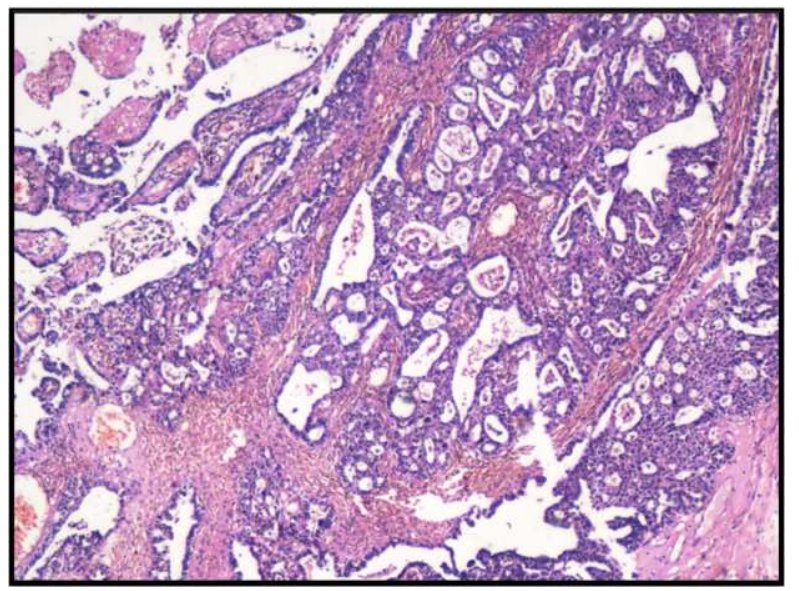

Fig. 3. HPE ( $H \& E$, X100) - Endometrial Adenocarcinoma type 2 -Section shows a tumor displaying features of serous papillary carcinoma

\section{Discussion}

Our study was conducted among women between 20-75 year old with history of abnormal uterine bleeding. Although The Bethesda system recommendation is 40 year, our consideration of wide age range was because shedding of normal endometrial cells has been associated with the following conditions: polyps, hyperplasias with and without
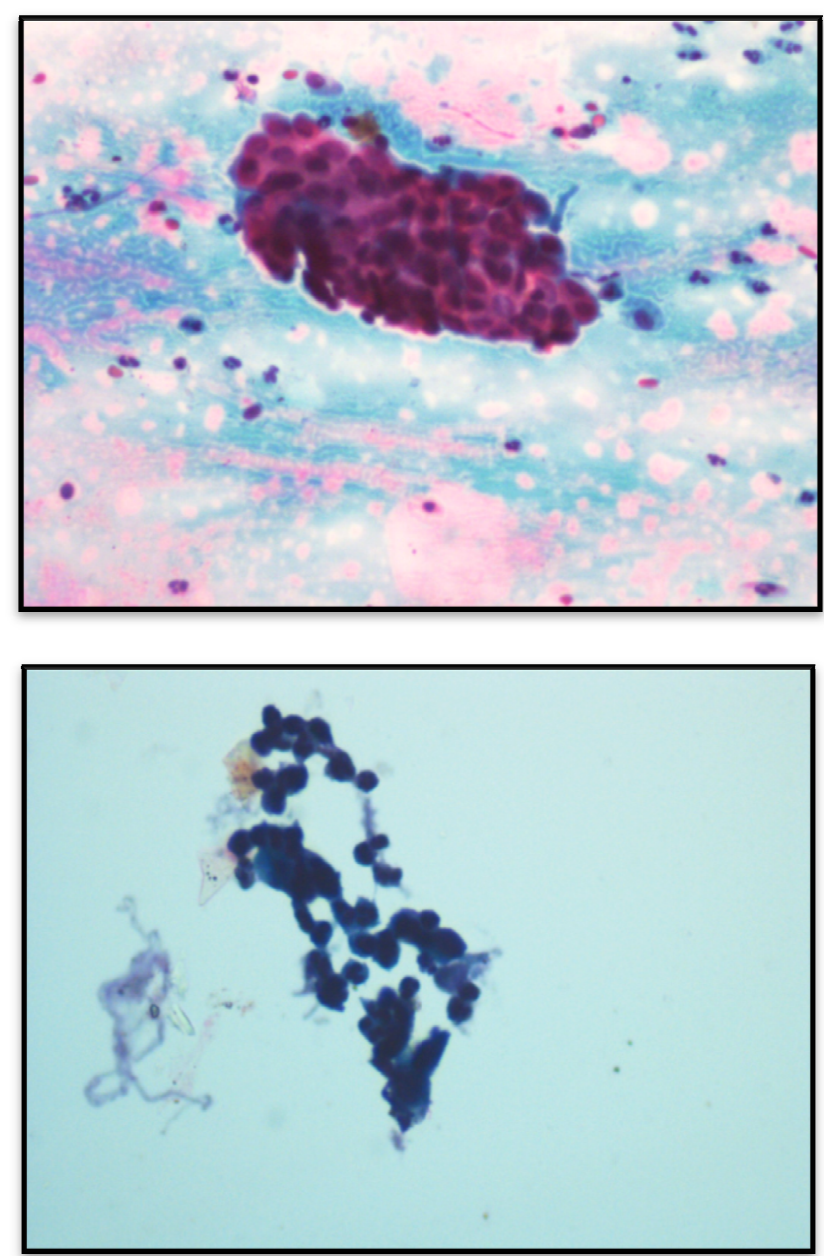

Fig. 4 \& 5. CPS \& MLBC (Pap, X400) Benign endometrial Cells - Smear shows endometrial cells in acinar pattern. Cells are small with round nuclei and scant cytoplasm

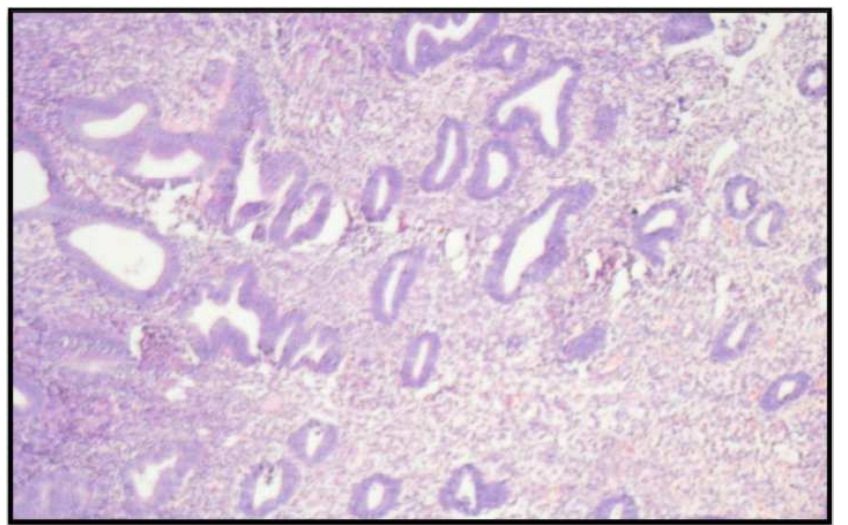

Fig. 6. $H P E(H \& E, X 100)$ - Proliferative Endometrium -Section shows round tubular endometrial gland and compact stroma

atypia, low and high grade adenocarcinomas, leiomyomata, atrophy, proliferative endometrium, immediate postpartum state, impending or early post abortion, acute endometritis, recent intrauterine instrumentation, IUD use, and cervical and vaginal endometriosis . ${ }^{14}$

This was also to include those high risk women under 40 who have obesity, type 2 diabetes, hypertension, anovulation/ Polycystic Ovary Syndrome (PCOS), and genetic factors 
such as defects in DNA mismatch repair genes, are at greater risk for a significant lesion if shedding endometrial cells out of phase. Studies with this stratification and sub analysis are lacking in the literature ${ }^{14}$.

In our study the youngest patient with endometrial carcinoma type 1 was 36 year, but no family history of uterine or breast malignancy was reported.

Unlike AEMCs and EMCCs, NEMCs are reported only in women 40 years or older based on The Bethesda System 2001 (TBS2001) because age is more consistently available than menopausal status or clinical symptoms and the incidence of significant endometrial pathology in women younger than 40 years is extremely low. ${ }^{15}$

Though studies have reported cancerous and precancerous endometrial lesions in postmenopausal women with benign endometrial cells in cytologic specimens ${ }^{16,17,18,19}$, our study showed only benign endometrial pathology.

Previous studies have suggested that reporting nEMCs in premenopausal women has little practical value. ${ }^{20,21}$ Another retrospective study found no significant difference in endometrial hyperplasia or malignancy when comparing women 40 years or older or 50 years or older with no endometrial cells with women with benign endometrial cells on Pap cytology. ${ }^{22}$

Our data also indicated that there was little significant pathology (cancerous / precancerous) in premenopausal women with benign endometrial cells on Pap cytology.

However, among 24 postmenopausal patients with atypical endometrial cells, six were diagnosed to have endometrial carcinoma and one person with complex hyperplasia with atypia on follow up.

Like our study Simsir et al ${ }^{23}$ and Kerpsack et al. study showed a significant correlation between postmenopausal bleeding with presence of endometrial cell in cervical cytology and endometrial pathology mainly endometrial carcinoma. $^{24}$

It is difficult to get an accurate rate of endometrial cells recognized on the Pap test because the total population studied is not defined in many studies. ${ }^{9}$ The age of the screened population, which affects the prevalence of endometrial cells found in that population, is often not clarified $^{25}$. Very few studies had clearly mentioned study group population.

Comparisons between those studies also were hampered by significant differences in the classification of Pap tests, including a lack of consensus about whether benignappearing endometrial cells constitute normal or abnormal findings. ${ }^{8}$

Regarding the method of collection, sensitivity of cervical cytology testing for true glandular neoplasia is less than that for squamous lesions, in part because glandular lesions arise in the endocervical canal and endometrial cavity where they may be difficult to sample.

But available literature suggested compared with conventional cytology, LBC may be associated with a higher prevalence of NECs because of more consistent use of sampling instruments for $\mathrm{LBC}$ with better access to this area. $^{7}$

However, we used manual method of liquid based preparation and found that the MLBC method was comparable to the CPS though our data showed no much difference in prevalence of endometrial cells between CPS and MLBC

Increased detection of cellular abnormalities by liquid based method depends on many factors like adequacy of sample, type of spatula used to collect sample and type of sampling whether direct to vial or split-sample method.

There are 3 possible explanations for improved sensitivity:

1. LBC improves sample collection by markedly increasing number of cells that leave collection device into vial.

2. LBC reduces obscuring elements and thus allows for detection of abnormal cells that could have been otherwise hidden on CPS.

3. Better preservation of abnormal cells on LBC slide allows for more definitive categorization of abnormal cells

\section{Conclusion}

Endometrial carcinoma early detection is becoming an increasingly important challenge. So the time has arrived to consider screening for endometrial pathology seriously.

Recently, developed countries like Japan has conducted randomized controlled trial comprising liquid based endometrial cytology as screening tool for the early detection of their considerately increasing endometrial cancer and gained a huge response.

In developing country specially in low resources set up, MLBC can be of little help. It overcomes the limitations of CPS like removing obscuring factors and increasing diagnostic accuracy. Also, future ancillary techniques like preparation of cell blocks, immunocytochemsitry and HPVtesting becomes possible in testing of new paradigms for screening strategies that are required in such settings.

From our study, we like to stress upon that-

1. presence of atypical endometrial cells in all women \& benign endometrial cells in postmenopausal women $(>40 \mathrm{yr})$ has considerable clinical implications \& further diagnostic evaluation for endometrial sampling is utmost important .

2. manual liquid based cytology as an alternative and more effective screening for endometrial pathology in lowresource settings

3. further need for additional trials to evaluate the diagnostic accuracy of liquid based endometrial cytology in the evaluation of endometrial pathology.

\section{References}

[1] Iatrakis G, Diakakis I, Kourounis G et al. Postmenopausal uterine bleeding. Clin Exp Obstet Gynecol 1970;19:61-70. 
[2] Nagele F, O'Connor H, Baskett TF et al. Hysteroscopy in women with abnormal uterine bleeding on hormone replacement therapy: a comparison with postmenopausal bleeding. Fertil Steril 1996;65:1145-50.

[3] Remondi C, Sesti F, Bonanno E, Pietropolli A, Piccione E. Diagnostic accuracy of liquid-based endometrial cytology in the evaluation of endometrial pathology in postmenopausal women.Cytopathology 2013;24:365-71.

[4] Vuento MH, Pirhonen JP, Makinen JI et al. Screening for endometrial cancer in asymptomatic postmenopausal women with conventional and colour Doppler sonography. $\mathrm{Br} \mathrm{J}$ Obstet Gynaecol 1999;106:348-53.

[5] Wu HH, Schuetz MJ, Cramer H. Significance of benign endometrial cells in Pap smears from postmenopausal women.J Reprod Med. 2001;46:795-798.

[6] Papanicolaou GN,Traut HF. Diagnosis of Uterine Cancer by the Vaginal Smear. New York, NY: The Commonwealth Fund; 1943.

[7] Canfell K, Kang YJ, Clements M, Moa AM, Barel V. Normal Endometrial Cells in Cervical Cytology: Systematic Review of Prevalence and Relation to Significant Endometrial Pathology .Journal of medical screening 2008; vol. 15 ( 4) : 188-98.

[8] Thrall M, Kjeldahl K, Gulbahce HE, Pambuccian SE. Liquidbased Papanicolaou test (SurePath) interpretations before histologic diagnosis of endometrial hyperplasias and carcinomas. Cancer Cytopathology 2007;111(4):217-23.

[9] Greenspan DL, Cardillo M ,Davey DD, Heller DS, Moriarty AT.Endometrial Cells in Cervical Cytology: Review of Cytological Features and Clinical Assessment . American Society for Colposcopy and Cervical Pathology Journal of Lower Genital Tract Disease2006; 10(2): 111-22.

[10] Buccoliero AM, Resta L, Napoli A, Taddei GL. Liquid based endometrial cytology: the Florence and Bari experience. Pathologica 2009;101:80-4.

[11] Norimatsu Y, Kouda H, Kobayashi TK et al. Utility of thinlayer preparations in the endometrial cytology: evaluation of benign endometrial lesions. Ann Diagn Pathol 2008;12:103-11.

[12] Alves VAF, Bibbo M, Schemitt FCL, Milanezi F, Filho AL. Comparison of manual and automated methods of liquidbased cytology a morphologic study. Acta Cytol 2004.48:187193.
[13] Lee, Kelly D, Gravitt PE, Fansler Matosem JA, Clark DP. Validation of a low-cost, liquid based screening method of cervical intraepithelial neoplasia. Am J Obstet and Gynecol 2006. 195,965-70.

[14] Ng AB, Reagan JW, Cechner RL. The precursors of endometrial cancer: a study of their cellular manifestations.Acta Cytol 1973;17:437-48.

[15] Zaibo American Society for Clinical PathologyAm J Clin Pathol 2012;138:79-84.

[16] Li Z., Gilbert C., Yang H., and Zhao C.Histologic Follow-up in Patients With Papanicolaou Test Findings of Endometrial (Cells Results From a Large Academic Women's Hospital Laboratory.

[17] $\mathrm{Wu} H H$, Schuetz MJ, Cramer H. Significance of benign endometrial cells in Pap smears from postmenopausal women.J Reprod Med. 2001;46:795-798.

[18] Montz FJ. Significance of 'normal endometrial cells in cervical cytology from asymptomatic postmenopausal women receiving hormone replacement therapy. Gynecol Oncol 2001;81:33-9.

[19] Brogi E, Tambouret R, Bell DA. Classification of benign endometrial glandular cells in cervical smears from postmenopausal women. Cancer. 2002;96:60-66.

[20] Karim BO, Burroughs FH, Rosenthal DL, et al. Endometrialtype cells in cervico-vaginal smears: clinical significance and cytopathologic correlates. Diagn Cytopathol. 2002;26:123- 127.

[21] Kapali M, Agaram NP, Dabbs D, et al. Routine endometrial sampling of asymptomatic premenopausal women shedding normal endometrial cells in Papanicolaou tests is not cost effective. Cancer. 2007;111:26-33.

[22] Thrall MJ, Kjeldahl KS, Savik K, et al. Significance of benign endometrial cells in papanicolaou tests from women aged $>$ or $=40$ years. Cancer. 2005;105:207-216.

[23] Simsir A, Carter W, Elgert P, Cangiarella J. Reportingendometrial cells in women 40 years and olderAssessing the clinical usefulness of Bethesda 2001. Am J Clin Pathol 2005;123:571-5.

[24] Ozkan F, Ramzy I, Mody DR. Glandular lesions of the cervix on thin-layer Pap tests. Validity of cytologic criteria used in identifying significant lesions. Acta Cytol 2004;48:372-379.

[25] Bean S, Connolly K, Roberson J, et al. Incidence and clinical significance of normal endometrial cells in patients 40 years and older (abstract). Acta Cytol 2004;48:677. 